ADVERSE DRUG REACTION

\title{
Warfarin and celecoxib interaction in the setting of cytochrome P450 (CYP2C9) polymorphism with bleeding complication
}

\author{
H Malhi, B Atac, A K Daly, S Gupta
}

Postgrad Med J 2004;80:107-109. doi: 10.1136/pmi.2003.006478

Drug metabolism may be perturbed by genetically determined differences in the metabolic activity of cytochrome P450 enzymes. The authors encountered extensive bleeding in a patient receiving warfarin for anticoagulation after the introduction of celecoxib, an anti-inflammatory drug. As the CYP2C9 enzyme metabolises these drugs, it was determined whether variant alleles were responsible for altering warfarin handling. Genetic analysis established that the patient was a compound heterozygote with CYP2C9*2 and *3 variant alleles, which exhibit lower drug metabolising capacity and enhance susceptibility to drug toxicity.

G enetic differences in the activity of cytochrome P450 (CYP) genes often account for variable drug metabolism. Many common drugs, including warfarin and non-steroidal anti-inflammatory drugs, are metabolised by the CYP2C9 enzyme. Three variants of CYP2C9 gene, designated $C Y P 2 C 9 * 1, * 2$ and $* 3$, are encountered in white people. ${ }^{1-3}$ The enzymatic activity of $* 2$ and $* 3$ variant genes is decreased significantly, and carriers of the $* 3$ variant are at risk for complications, such as bleeding after use of warfarin in small amounts. $^{23}$ Recently, inflammatory drugs that work by inhibiting cyclo-oxygenase (COX)-2 activity attracted interest. ${ }^{4}$ For instance, COX-2 inhibitors were thought to have less potential for causing gastrointestinal bleeding. The COX-2 inhibitor, celecoxib, is primarily metabolised by the CYP2C9 enzyme, ${ }^{5}$ and is being increasingly popular. In a limited sample of patients, celecoxib was not found to alter the pharmacokinetics of warfarin. ${ }^{6}$ However, whether this group of subjects included any with variant CYP2C9 alleles, which might have resulted in altered pharmacokinetics of warfarin, was unknown. Our experience suggests that celecoxib may perturb warfarin metabolism, leading to greater anticoagulation in the presence of variant CYP2C9 genes.

\section{CASE REPORT}

An elderly woman developed ecchymoses with a decline in haemoglobin concentration from 160 to $85 \mathrm{~g} / \mathrm{l}$ over one week. She was receiving $2.5 \mathrm{mg}$ warfarin daily after a pacemaker one year previously. The international normalised ratio (INR) of prothrombin time on this dose of warfarin ranged from 2.2 to 2.6. She was given celecoxib four weeks previously for pain in multiple joints. This resulted in prolongation of prothrombin time within two weeks with the INR increasing to 3.5 and the dose of warfarin was decreased to $1 \mathrm{mg}$ daily. The other routine medications included digoxin, ranitidine, and atorvastatin. Her diet was unchanged; her bowel habits were also unchanged.
Physical examination showed that vital signs were stable. Extensive ecchymotic areas involved both flanks, left upper limb, under breasts, perineal area, and posterior thorax. There was no oedema, arthritis, stigmata of chronic liver disease, or evidence for vasculitis. Cardiac sounds were normal without significant murmurs, chest was clear, the abdomen was soft and non-tender, without liver or spleen enlargement. No focal neurological deficits were found. The INR was $>10$. Blood urea nitrogen, serum creatinine, and liver tests were normal. Computed tomography of the abdomen excluded internal haemorrhage.

The coagulation abnormality resolved completely one month after celecoxib and warfarin were discontinued. The patient made a full recovery.

\section{ANALYSIS OF CYP2C9 POLYMORPHISM}

These studies followed the guidelines established by the institutional review board and informed consent was obtained from the patient. Genomic DNA was extracted from whole blood and an established human liver cell line, HepG2 (DNA Extractor WB Kit, Wako Chemicals USA, Inc, Richmond, VA). Reference CYP2C9 variant DNAs were available (collection of Dr A K Daly). CYP2C9 primers were according to Sullivan-Klose et al. ${ }^{7}$ A $50 \mu$ polymerase chain reaction (PCR) used $2.5 \mathrm{mM}$ magnesium chloride, $1.2 \mu \mathrm{M}$ primers, $1 \mu \mathrm{g}$ genomic DNA, and $0.5 \mathrm{U}$ DNA polymerase (AmpliTaq, PE Biosystems, Foster City, CA) with 35 cycles $\left(94^{\circ} \mathrm{C}, 5 \mathrm{~min} ; 94^{\circ} \mathrm{C}, 60 \mathrm{~s} ; 55^{\circ} \mathrm{C}, 90 \mathrm{~s} ; 72^{\circ} \mathrm{C}, 30 \mathrm{~s}\right.$; and $72^{\circ} \mathrm{C}$, $7 \mathrm{~min})$. PCR products were digested with Ava II or Kpn I (Promega Corp, Madison, WI) and resolved in $1 \%$ agarose containing ethidium bromide. The CYP $2 C 9 * 2$ allele loses an Ava II site and the *3 allele gains a Kpn I site. ${ }^{7}$

\section{*2 Variant analysis}

All samples showed the expected 691 base pair (bp) band. Reference DNAs $\left(* 1 /{ }^{*} 1\right.$ and $\left.{ }^{*} l / * 3\right)$ produced 527 and $164 \mathrm{bp}$ Ava II fragments (fig 1A). Ava II digestion of $* 1 / * 2$ product produced 691, 527, and 164 bp bands. Our patient showed 691, 527 and 164 bp Ava II fragments, indicating an *2 allele (lane 9, fig 1A).

\section{*3 Variant analysis}

All samples showed the expected $141 \mathrm{bp}$ product (fig 1B). Kpn I did not digest ${ }^{*} 1 /{ }^{*} 1,{ }^{*} 1 /{ }^{*} 2$, and HepG2 DNAs, whereas $* 1 / * 3$ reference and test DNAs were partially digested with 141, 111 and $30 \mathrm{bp}$ fragments (lanes 7 and 9, fig 1B).

Abbreviations: bp, base pair; COX, cyclo-oxygenase; INR, international normalised ratio; PCR, polymerase chain reaction 


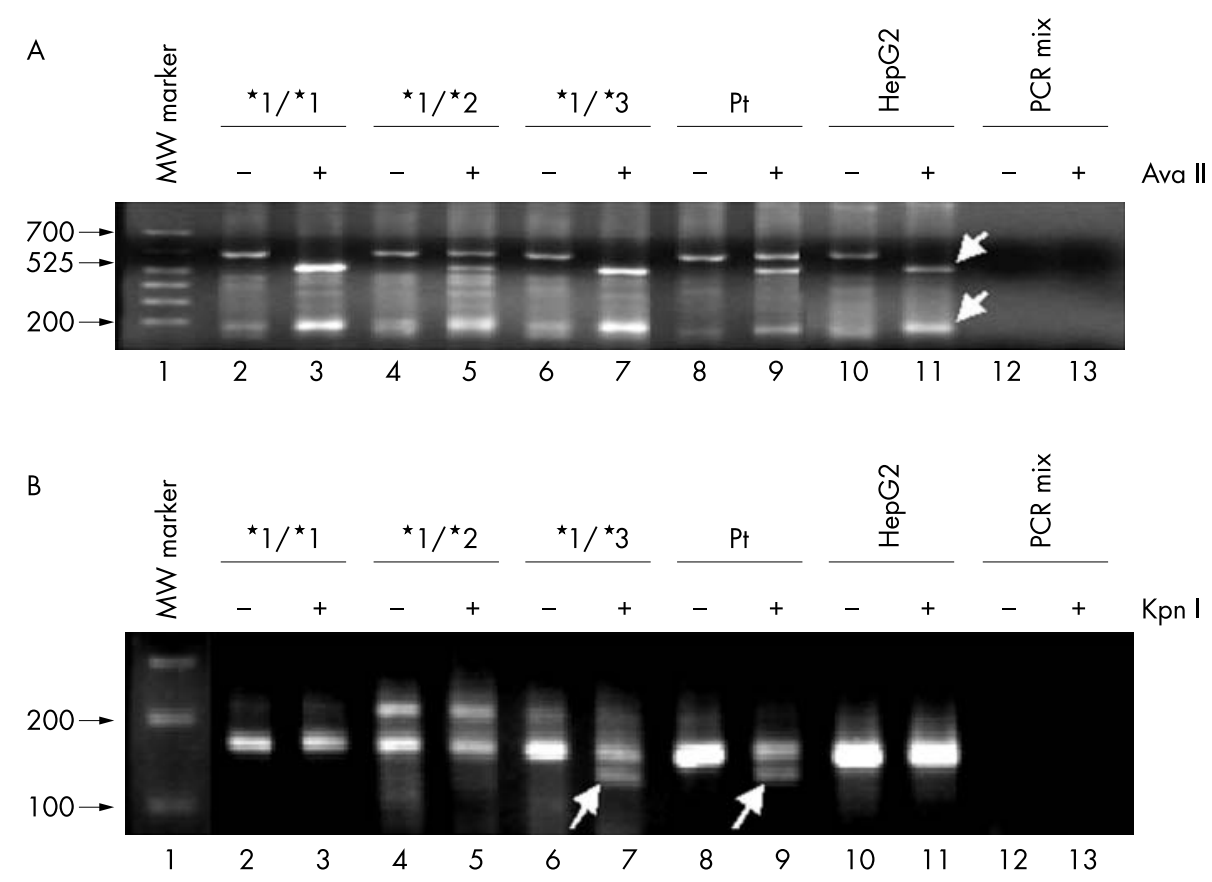

Figure 1 PCR of CYP2C9 alleles showing analysis of *2 variant (A) and *3 variant alleles (B). Lane 1, molecular weight marker; lanes 2 and $3,{ }^{*} 1 /{ }^{*} 7$ reference DNA; lanes 4 and $5,{ }^{*} 1 /{ }^{*} 2$ reference DNA; lanes 6 and $7,{ }^{*} 7 /{ }^{*} 3$ reference DNA; lanes 8 and 9 , DNA from patient; lanes 10 and 11, DNA from HepG2 cells; and lanes 12 and 13, PCR mix alone. Lanes marked by (+) show DNA digested by Ava II or Kpn I. Arrows in panel A show 527 and 164 bp * $1 /{ }^{*} 1$ fragments. Arrows in (B) show 111 bp *3 fragment. The 30 bp *3 fragment is not shown.

Sequence analysis verified *2 and *3 CYP2C 9 alleles in the patient.

\section{DISCUSSION}

This patient was a compound $* 2 / * 3$ CYP2C9 heterozygote. Despite the lower warfarin dose of $1 \mathrm{mg}$ daily, competition between celecoxib and warfarin led to severe anticoagulation and bleeding. These findings are quite compatible with warfarin and celecoxib interaction. Such an interaction has been suspected relatively rarely. ${ }^{8}$ We focused on CYP2C9 because this pathway inactivates both warfarin and celecoxib, accounting for $\sim 80 \%$ of drug clearances, whereas $* 2$ and $* 3$ variants possess only $\sim 12 \%$ and $5 \%$ enzyme activity, which should increase drug retention. ${ }^{10-12}$

Aithal et $a l^{2}$ and Taube et $a l^{3}$ established that markedly lower warfarin doses were sufficient for anticoagulation in *2 and *3 CYP2C9 carriers (average $<1.5 \mathrm{mg} v 5 \mathrm{mg}$ warfarin daily). ${ }^{3}$ Moreover, warfarin maintenance in compound CYP 2 C $9 * 2 / * 3$ heterozygotes was $82 \%$ of the dose required by ${ }^{*} 1 /{ }^{*} 1$ CYP2C9 carriers. Although overanticoagulation rates during warfarin maintenance with variant CYP2C9 alleles

\section{Summary points}

- Patients receiving drugs metabolised by the same cytochrome P450 system could be at risk for drug interactions.

- Presence of variant cytochrome P450 genes with lower enzymatic activity increases susceptibility for drug interactions.

- This case demonstrates the following specific points:

- Metabolism of warfarin was perturbed and led to bleeding in the presence of variant CYP2C9 genes by coadministration of celecoxib, which is a COX-2 inhibitor.

- Analysis of potential interactions between warfarin and other drugs sharing metabolic pathways should include the context of CYP2C9 polymorphisms. were (not surprisingly) similar, ${ }^{3}$ our patient indicates that drug interactions can obviously alter this situation. Celecoxib metabolism decreases up to $90 \%$ with $* 2$ and $* 3$ variant alleles and celecoxib exhibits high CYP2C9 affinity. ${ }^{12}{ }^{12}$ These perturbations could delay or prevent warfarin metabolism, leading to gradual warfarin accumulation and delayed bleeding, as seen in our patient.

The CYP2C $9{ }^{*} I /{ }^{*} I$ allele was encountered most frequently $(\sim 80 \%)$ in European populations. ${ }^{13}$ However, ${ }^{*} 2$ and $* 3$ alleles were prevalent in $11 \%$ and $5 \%-7 \%$, respectively, of these populations, which is not insignificant. Therefore, many patients will be at risk for drug interactions mediated by CYP2C9 variants. Use of warfarin for anticoagulation, as well as celecoxib and related drugs, for arthritis and/or other conditions will rise further in the older age groups and thus it will be appropriate to consider the type of drug interaction seen in our patient. This suggests that suitable ways to define the risk for such complications in large patient populations should be examined. For example, the value of prospective CYP2C9 genotyping needs to be addressed in people requiring long term anticoagulation, since single nucleotide polymorphisms are amenable to high throughput testing, which should make such an approach practical.

\section{Authors' affiliations}

H Malhi, Marion Bessin Liver Research Center and Department of Medicine, Albert Einstein College of Medicine, New York, USA

B Atac, Department of Medicine, Albert Einstein College of Medicine, New York, USA

A K Daly, Department of Pharmacological Sciences, University of Newcastle Medical School, Newcastle upon Tyne, UK

S Gupta, Marion Bessin Liver Research Center and Departments of Medicine and Pathology, Albert Einstein College of Medicine, New York, USA

Correspondence to: Dr Sanjeev Gupta, Albert Einstein College of Medicine, Ullmann Building, Room 625, 1300 Morris Park Avenue, Bronx, New York 10461, USA; sanjvgupta@pol.net

Submitted 14 February 2003

Accepted 26 March 2003 


\section{REFERENCES}

1 Yasar U, Eliasson E, Dahl ML, et al. Validation of methods for CYP2C9 genotyping: frequencies of mutant alleles in a Swedish population. Biochem Biophys Res Commun 1999;254:628-31.

2 Aithal GP, Day CP, Kesteven PJ, et al. Association of polymorphisms in the cytochrome P450 CYP2C9 with warfarin dose requirement and risk of bleeding complications. Lancet 1999;353:717-9.

3 Taube J, Halsall D, Baglin T. Influence of cytochrome P-450 CYP2C9 polymorphisms on warfarin sensitivity and risk of over-anticoagulation in patients on long-term treatment. Blood 2000;96:1816-9.

4 FitzGerald GA, Patrono C. The coxibs, selective inhibitors of cyclooxygenase2. N Engl J Med 2001;345:433-42.

5 Davies NM, McLachlan AJ, Day RO, et al. Clinical pharmacokinetics and pharmacodynamics of celecoxib: a selective cyclo-oxygenase- 2 inhibitor. Clin Pharmacokinet 2000;38:225-42.

6 Karim A, Tolbert D, Piergies A, et al. Celecoxib does not significantly alter the pharmacokinetics or hypoprothrombinemic effect of warfarin in healthy subjects. J Clin Pharmacol 2000;40:655-63.
7 Sullivan-Klose TH, Ghanayem BI, Bell DA, et al. The role of the CYP2C9Leu359 allelic variant in the tolbutamide polymorphism. Pharmacogenetics 1996;6:341-9.

8 Linder JD, Monkemuller KE, Davis JV, et al. Cyclooxygenase-2 inhibitor celecoxib: a possible cause of gastropathy and hypoprothrombinemia. South Med J 2000;93:930-2.

9 Mersfelder TL, Stewart LR. Warfarin and celecoxib interaction. Ann Pharmacother 2000;34:325-7.

10 Tang C, Shou M, Rushmore TH, et al. In-vitro metabolism of celecoxib, a cyclooxygenase- 2 inhibitor, by allelic variant forms of human liver microsomal cytochrome P450 2C9: correlation with CYP2C9 genotype and in-vivo pharmacokinetics. Pharmacogenetics 2001;11:223-5

11 Crespi CL, Miller VP. The R144C change in the CYP2C9*2 allele alters interaction of the cytochrome P450 with NADPH:cytochrome P450 oxidoreductase. Pharmacogenetics 1997;7:203-10.

12 Tang C, Shou $M$, Mei $Q$, et al. Major role of human liver microsomal cytochrome P450 2C9 (CYP2C9) in the oxidative metabolism of celecoxib, a novel cyclooxygenase-ll inhibitor. J Pharmacol Exp Ther 2000;293:453-9. 